\title{
Exosomal microRNA-I 4 I is upregulated in the serum of prostate cancer patients
}

This article was published in the following Dove Press journal:

OncoTargets and Therapy

31 December 2015

Number of times this article has been viewed

\author{
Zhuo Li ${ }^{1,2, *}$ \\ Yue-Yun Ma ${ }^{1}, *$ \\ Juan Wang' \\ Xian-Fei Zeng' \\ Rui $\mathrm{Li}^{\prime}$ \\ Wei Kang ${ }^{2}$ \\ Xiao-Ke Hao' \\ 'Department of Laboratory Medicine, \\ Xijing Hospital, Fourth Military \\ Medical University, ${ }^{2}$ Department \\ of Laboratory Medicine, The First \\ Affiliated Hospital of Xi'an Medical \\ University, Xi'an, Shaanxi Province, \\ People's Republic of China \\ *These authors contributed equally \\ to this work
}

Correspondence: Xiao-Ke Hao Department of Laboratory Medicine, Xijing Hospital, Fourth Military Medical University, I5 Changle West Road, Xi'an 7I0032, Shaanxi Province, People's Republic of China

Tel +862984775572

Email haoxkg@126.com
Purpose: Novel biomarkers for the diagnosis of prostate cancer $(\mathrm{PCa})$ are urgently required. Increasing evidence suggests that exosomal microRNAs (miRNAs or miRs) in serum may be potential noninvasive biomarkers for certain diseases. The objective of the present study was to investigate and assess whether exosomal miR-141 is an effective biomarker for human PCa.

Methods: In the present study, exosomes were isolated from the serum of patients with $\mathrm{PCa}$, patients with benign prostate hyperplasia $(\mathrm{BPH})$, and healthy volunteers. The total RNA was extracted from the exosomes and the level of miR-141 was analyzed by quantitative reverse transcription-polymerase chain reaction. The expression levels of $m i R-141$ were compared between the whole serum and the serum exosomes of the three groups. Subsequently, the relevance of the exosomal expression of $m i R-141$ to the clinicopathological factors in PCa was investigated.

Results: The expression of $m i R-141$ was higher in exosomes compared with whole serum (control group, $P=0.0003$; $\mathrm{BPH}$ group, $P=0.0016$; $\mathrm{PCa}$ group, $P<0.0001)$. The level of serum exosomal $m i R-141$ was significantly higher in the patients with $\mathrm{PCa}$ compared with the patients with $\mathrm{BPH}$ and the healthy controls ( 3.85 -fold, $P=0.0007$ and 4.06 -fold, $P=0.0005$, respectively). In addition, the expression levels were significantly higher in metastatic PCa compared with localized PCa $(P<0.0001)$. Receiver-operating characteristic curve revealed that the serum exosomal miR-141 yielded an area under the curve of 0.8694 , with $80 \%$ sensitivity and $87.1 \%$ specificity in discriminating patients with metastatic PCa from the patients with localized PCa.

Conclusion: Serum exosomes may serve as a more suitable material compared with the whole serum for measuring circulating $m i R-141$ levels in patients with $\mathrm{PCa}$. Exosomal miR-141 is upregulated in the serum from patients with $\mathrm{PCa}$ compared with patients with $\mathrm{BPH}$ or the healthy volunteers, and it may be a useful potential biomarker for the diagnosis of metastatic PCa.

Keywords: exosomes, microRNA-141, serum, prostate cancer, biomarker

\section{Introduction}

Prostate cancer $(\mathrm{PCa})$ is one of the most common male malignancies and the second leading cause of male cancer-associated mortality. ${ }^{1}$ In the People's Republic of China, the incidence and mortality rates of $\mathrm{PCa}$ are clearly increasing. In addition, Chinese patients with PCa exhibit a higher proportion $(13.3 \%-26 \%)$ of bone metastasis in the initial diagnosis. ${ }^{2-4}$ Currently, prostate-specific antigen (PSA) is the commonly used biomarker for $\mathrm{PCa}$, which is measured in the blood and exhibits increased levels in patients with PCa. However, limitations of PSA screening usually lead to overdiagnosis and overtreatment. ${ }^{5}$ Therefore, novel biomarkers with increased specificity for the diagnosis and prognosis of PCa are urgently required. Increasing evidence suggests that exosomal microRNAs (miRNAs or miRs) in circulating fluids may offer an attractive potential as noninvasive biomarkers for certain diseases, including cancer. ${ }^{6-8}$

Exosomes are 30-100 nm membrane vesicles, which are released from numerous cell types into the extracellular space. Exosomes are widely distributed in the 
blood, urine, and other bodily fluids. Lipids and proteins are the predominant components of exosomal membranes. ${ }^{9}$ Exosomes are important in the exchange of information between cells. ${ }^{10}$ Previously, miRNAs have been identified in exosomes, which can be taken up by neighboring or distant cells, and subsequently modulate the recipient cells. ${ }^{11}$ Additionally, several previous studies have revealed that stable miRNAs can be detected in exosomes, as well as in serum, and the majority of serum-circulating miRNAs are enriched in exosomes. Furthermore, the quantity and composition of exosomal miRNAs are different between patients and healthy individuals. ${ }^{12,13}$ Therefore, exosomal miRNAs may serve as valuable noninvasive biomarkers for the diagnosis and prognosis of certain diseases.

However, a number of previous studies addressing the expression levels of circulating miRNAs in clinical patients with PCa were performed on total serum or plasma samples. ${ }^{14-17}$ With regards to exosomal miRNAs, to the best of our knowledge, only a few previous studies have reported the use of exosomal miRNAs for prognosis in patients with PCa. ${ }^{18}$ In addition, whether there is a potential benefit in using miRNAs in exosomes compared with in the total serum as biomarkers for patients with PCa remains to be elucidated.

miR-141 is one of the most common cancer-associated miRNAs, which has been reported as tumor suppressor in several malignancies, such as gastric cancer, ${ }^{19,20}$ pancreatic cancer, ${ }^{21}$ breast cancer, ${ }^{22,23}$ renal cell carcinoma, ${ }^{24,25}$ hepatocellular carcinoma, ${ }^{26}$ and so on. On the contrary, increasing evidence shows that miR-141 is upregulated in PCa, which is correlated with a high Gleason score or metastasis of patients. ${ }^{14,27-30}$ Therefore, $m i R-141$ is considered as a potential miRNA biomarker for PCa. However, the comparison of the expression levels of $m i R-141$ in serum samples and exosomes isolated from the serum of patients with $\mathrm{PCa}$ remains to be elucidated. In the present study, the expression levels of miR-141 in exosomes isolated from the serum samples of patients with $\mathrm{PCa}$, benign prostate hyperplasia $(\mathrm{BPH})$, and healthy volunteers were investigated. Furthermore, the potential clinical value of using exosomal $m i R-141$ as a biomarker for the detection of PCa was assessed.

\section{Materials and methods Patients and blood samples}

Patients initially diagnosed with PCa (no prior treatment) at Xijing Hospital of Fourth Military Medical University (Xi'an, People's Republic of China) between January 2013 and December 2014 were included in the PCa group. Patients with newly diagnosed BPH (no prior treatment) were enrolled in the BPH group. Age- and sex-matched healthy individuals were collected as a control group. A serum versus exosomes cohort (20 patients with $\mathrm{PCa}, 20$ patients with $\mathrm{BPH}$, and 20 control volunteers) (Table 1) and a follow-up exosomes cohort (51 patients with PCa and 40 control volunteers) (Table 2) were included in the present study. Clinical and biochemical features such as age, serum PSA, Gleason score, clinical stage, and metastasis of the patients were recorded in detail. PCa diagnosis was confirmed by histological examination or needle biopsy detected. Histopathologic Gleason score was performed based on the criteria of the World Health Organization, and classified as well differentiated (Gleason score $\leq 6$ ), moderately differentiated (Gleason score $=7$ ), and poorly differentiated/undifferentiated (Gleason score $\geq 8$ ). Meanwhile, tumors were staged according to the seventh edition (2010) of the American Joint Committee on CancerCancer Staging Manual. T-classification of PCa was divided as organ-confined (T1/T2) or nonorgan-confined (T3/T4). ${ }^{31}$

Table I Clinical characteristics of participants (serum vs exosomes cohort)

\begin{tabular}{|c|c|c|c|c|}
\hline Characteristic & PCa patients & BPH patients & Healthy controls & $P$-value \\
\hline Total number & 20 & 20 & 20 & \\
\hline Age (years), median (IQR) & $69.50(63.25-78.75)$ & $70.00(64.25-75.75)$ & $72.50(66.25-75.00)$ & 0.8029 \\
\hline PSA (ng/mL), median (IQR) & $25.83(7.29-189.50)$ & $7.64(2.53-10.64)$ & I.II (0.7I-2.3I) & $<0.000 I^{* *}$ \\
\hline \multicolumn{5}{|l|}{ Gleason score } \\
\hline$\leq 6$ & 4 & & & \\
\hline 7 & 10 & & & \\
\hline$\geq 8$ & 6 & & & \\
\hline \multicolumn{5}{|l|}{ Clinical stage } \\
\hline $\mathrm{TI} / \mathrm{T} 2$ & 15 & & & \\
\hline T3/T4 & 5 & & & \\
\hline \multicolumn{5}{|l|}{ Metastasis } \\
\hline No & 14 & & & \\
\hline Yes & 6 & & & \\
\hline
\end{tabular}

Note: $* * P<0.01$.

Abbreviations: BPH, benign prostate hyperplasia; IQR, interquartile range; PCa, prostate cancer; PSA, prostate-specific antigen. 
Table 2 Clinical characteristics of participants (follow-up exosomes cohort)

\begin{tabular}{llll}
\hline Characteristic & PCa patients & Healthy controls & P-value \\
\hline Total number & 51 & 40 & $70.50(65.00-74.75)$ \\
Age (years), median (IQR) & $70.00(64.00-76.00)$ & $1.03(0.70-1.36)$ & \\
PSA (ng/mL), median (IQR) & $57.0 I(14.33-196.00)$ & \\
Gleason score & & \\
$\quad \leq 6$ & 8 & \\
7 & 23 & \\
$\geq 8$ & 20 & \\
Clinical stage & & \\
TI/T2 & 24 & \\
T3/T4 & 27 & \\
Metastasis & & \\
No & 31 & \\
Yes & 20 & \\
\hline
\end{tabular}

Note: $* * P<0.01$.

Abbreviations: IQR, interquartile range; PCa, prostate cancer; PSA, prostate-specific antigen.

Blood sampling and examination were performed prior to treatment, including surgery, radiotherapy, or chemotherapy. The peripheral blood was collected into serum-separator tubes and allowed to stand for 1 hour at room temperature, prior to centrifugation at $3,000 \times g$ for 10 minutes. The resulting serum was transferred into fresh tubes and stored at $-80^{\circ} \mathrm{C}$ for further analysis. The present study was approved by the Medical Ethics Committee of Xijing Hospital of Fourth Military Medical University, and written informed consent was obtained from all participants (No XJYYLL-2015129).

\section{Exosome isolation}

Exosomes were extracted from serum samples using ExoQuick Exosome Precipitation Solution (System Biosciences, Mountain View, CA, USA), according to the manufacturer's instructions. Briefly, the serum was thawed on ice and centrifuged at $3,000 \times g$ for 15 minutes to remove cells and cell debris. The supernatant was filtered through a $0.22 \mu \mathrm{m}$ pore polyvinylidene fluoride filter (EMD Millipore, Billerica, MA, USA). Subsequently, one-fourth volume of ExoQuick solution was added to the supernatants and the samples were incubated at $4^{\circ} \mathrm{C}$ for 30 minutes, followed by centrifugation at $1,500 \times \mathrm{g}$ for 30 minutes. The final pellets, containing exosomes, were collected for characterization and RNA isolations (Figure S1).

\section{Transmission electron microscopy}

The exosomal pellets were resuspended in $50 \mu \mathrm{L}$ phosphatebuffered saline and a drop of the suspension was placed on a sheet of parafilm. A copper grid was floated on the drop for 2 minutes at room temperature and was subsequently touched onto a drop of $2 \%$ phosphotungstic acid and stained for 2 minutes. The grid was allowed to dry for several minutes and was examined. The images were captured using a Tecnai G2 Spirit electron microscope (FEI Co., Hillsboro, OR, USA).

\section{Western blot}

The total proteins were extracted from the serum exosomal pellets using radioimmunoprecipitation assay lysis buffer (50 mmol/L Tris (pH 7.4), $150 \mathrm{mmol} / \mathrm{L} \mathrm{NaCl}, 1 \%$ Triton $\mathrm{X}-100,1 \%$ sodium deoxycholate, $0.1 \%$ sodium dodecyl sulfate) and quantified using a bicinchoninic acid protein assay (Thermo Fisher Scientific, Tokyo, Japan). Equivalent amounts of protein $(25 \mu \mathrm{g})$ from exosomes and exosomedepleted serum supernatant were separated by $12 \%$ sodium dodecyl sulfate polyacrylamide gel electrophoresis and transferred by electroblotting onto nitrocellulose membranes (Life Technologies, Carlsbad, CA, USA). Subsequently, the membranes were blocked for 1 hour at room temperature with blocking buffer (LI-COR Biosciences, Lincoln, NE, USA), and incubated overnight at $4{ }^{\circ} \mathrm{C}$ with rabbit antihuman heat shock protein 70 antibody and CD63 antibody $(1: 1,000$; RayBiotech, Norcross, GA, USA). $\beta$-Actin (1:1,000; Abcam, Cambridge, MA, USA) was used as loading control. After washing three times in Tris-buffered saline-Tween, the membranes were then incubated for 1 hour at room temperature with conjugated goat antirabbit immunoglobulin $\mathrm{G}$ (1:5,000; LI-COR Biosciences). The immunoreactive bands were visualized using an Odyssey Imaging system (LI-COR Biosciences).

\section{Fluorescent-activated cell sorting}

Fluorescent-activated cell sorting analysis of the serum exosomes was performed, as described previously. ${ }^{32}$ Briefly, the exosomes isolated from the serum were conjugated with $4 \mu \mathrm{m}$ aldehyde/sulfate latex beads (Invitrogen Life Technologies), 
followed by staining with phycoerythrin mouse antihuman CD63 or isotype control (BD Biosciences, San Jose, CA, USA). The analysis was performed using a BD FACScan flow cytometer (BD Biosciences).

\section{Quantitative reverse transcription- polymerase chain reaction}

Exosomal pelleted fraction isolated from $400 \mu \mathrm{L}$ serum was resuspended in $400 \mu \mathrm{L}$ nuclease-free water. Then, the total RNA containing miRNA was extracted from the exosomal pelleted suspensions or $400 \mu \mathrm{L}$ whole serum using an miRNeasy Serum/Plasma kit (Qiagen, Hilden, Germany) with the final elution volume of $15 \mu \mathrm{L}$. In the RNA isolation step, a total of $2 \mu \mathrm{L}$ synthetic Caenorhabditis elegans celmiR-39 (RiboBio, Guangzhou, the People's Republic of China) was added to each sample as a spike-in control. ${ }^{27,30}$ Quantitative reverse transcription-polymerase chain reaction (qRT-PCR) was performed using the PrimeScript RT Reagent kit and SYBR Premix Ex Taq kit (Takara Bio, Inc., Shiga, Japan). The reverse transcription reaction was carried out in $10 \mu \mathrm{L}$ containing $2 \mu \mathrm{L}$ of $5 \times$ PrimeScript buffer, $0.5 \mu \mathrm{L}$ of PrimeScript RT enzyme mix, $0.5 \mu \mathrm{L}$ of genespecific primer, and $7 \mu \mathrm{L}$ of RNA extract. Reaction mixtures were incubated at $37^{\circ} \mathrm{C}$ for 15 minutes, $85^{\circ} \mathrm{C}$ for 5 seconds and then held at $4^{\circ} \mathrm{C}$. Next, $2 \mu \mathrm{L}$ of complementary DNA was amplified with $10 \mu \mathrm{L}$ of $2 \times$ SYBR Premix Ex Taq, $0.8 \mu \mathrm{L}$ of gene-specific primers, $0.4 \mu \mathrm{L}$ of $50 \times \mathrm{ROX}$ reference dye II, and $6.8 \mu \mathrm{L}$ of nuclease-free water in a final volume of $20 \mu \mathrm{L}$. The qRT-PCR was run on an ABI 7500 Fast Detection system (Applied Biosystems, Foster City, CA, USA). The reaction mixtures were incubated at $95^{\circ} \mathrm{C}$ for 30 seconds, followed by 40 cycles at $95^{\circ} \mathrm{C}$ for 3 seconds and at $60^{\circ} \mathrm{C}$ for 30 seconds. At the end of the PCR cycles, melting curve analyses were performed to confirm the specificity of the PCR products. All the reactions were performed in triplicate. The bulge-loop qRT-PCR primers for miR-141 and cel-miR-39 were purchased from RiboBio. The relative expression levels of $m i R-141$ were normalized against cel-miR-39 using the comparative $2^{-\Delta \Delta C t}$ method. ${ }^{33}$

\section{Statistical analysis}

The miR-141 levels in the serum and the serum exosomal samples obtained from the same patients were compared using the Wilcoxon rank sum test. The Mann-Whitney $U$-test or Kruskal-Wallis test was used to determine the statistical significance of the miR-141 levels in different samples. Correlation analysis was performed using the Spearman test. The area under the curve (AUC) was calculated through a receiver-operating characteristic (ROC) curve analysis to determine the sensitivity and specificity of exosomal miR-141 as a diagnostic biomarker for metastatic PCa. Statistical analyses were performed using SPSS statistical software package (version 17.0; SPSS Inc., Chicago, IL, USA). $P<0.05$ was considered to indicate a statistically significant difference.

\section{Results}

\section{Exosomes are successfully isolated from human serum samples}

To confirm the quality of the serum exosomes isolated using ExoQuick, the serum exosomes were characterized by morphological and protein marker analyses. Transmission electron microscopy analysis revealed that the microvesicle clusters isolated from serum were round or oval membrane vesicles with the size predominantly between 30 and $100 \mathrm{~nm}$, and were homogeneous in appearance (Figure 1A). Western blot revealed that the specific exosomal protein markers, heat shock protein 70 and CD63, were expressed in isolated serum exosomal pellets as specific bands, but not in exosomedepleted serum supernatant (Figure 1B). Flow cytometric analysis also indicated that the isolated microvesicle clusters expressed CD63 (Figure 1C). These results demonstrated that the isolated serum particles exhibited characteristics of exosomes and confirmed the successful isolation of exosomes from human serum samples.

\section{Expression of miR-I $4 I$ is significantly higher in exosomes compared with in serum}

The expression of miR-141 was determined in whole serum samples and serum exosomal pellets isolated from the same serum in a cohort of 20 patients with $\mathrm{PCa}, 20$ patients with $\mathrm{BPH}$, and 20 healthy individuals (Table 1). The qRT-PCR results revealed that, in the three groups, the expression levels of exosomal miR-141 were all significantly higher compared with that in the serum circulating miR-141 (control group, $P=0.0003$; BPH group, $P=0.0016$; $P C a$ group, $P<0.0001$; Figure 2A). Furthermore, there were significant associations in the expression levels of $m i R-141$ between the whole serum and the exosomes (control group, $r=0.7474$ and $P=0.0002$; BPH group, $r=0.7368$ and $P=0.0002$; PCa group, $r=0.6692$ and $P=0.0013$ ).

In addition, the expression level of exosomal miR-141 was compared among the three groups. The results indicated that the expression of exosomal $m i R-141$ in patients with $\mathrm{PCa}$ was significantly higher compared with that of the patients with BPH and the healthy controls (3.85-fold, $P=0.0007$ and 4.06-fold, $P=0.0005$, respectively; Figure $2 \mathrm{~B}$ ). Similar results were observed in the whole serum samples; however, this 

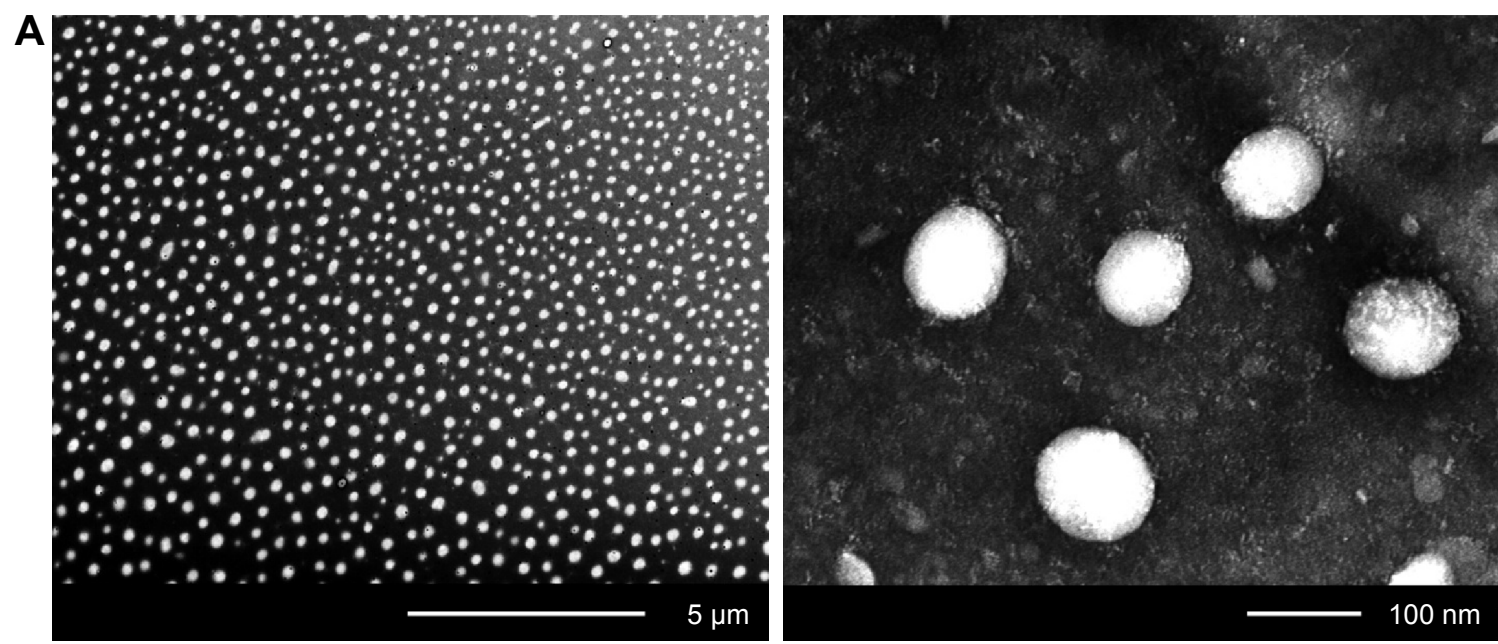

B

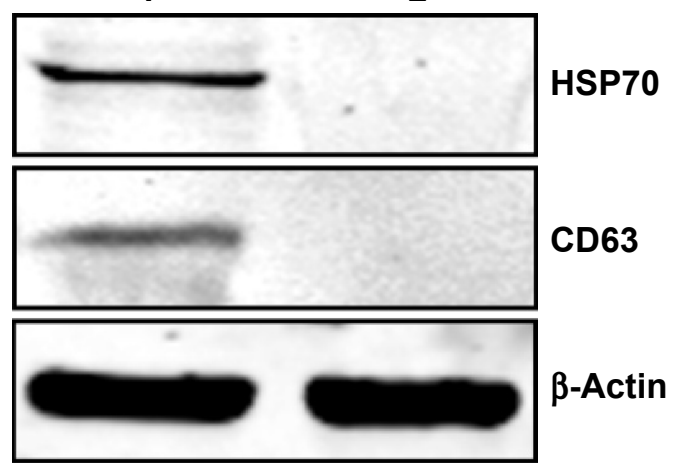

C

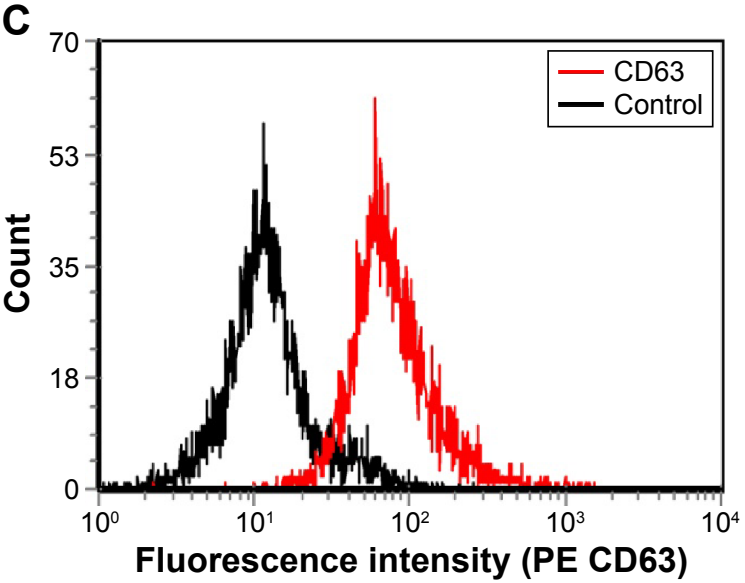

Figure I Characterization of isolated serum exosomes.

Notes: (A) Exosomes isolated from serum samples were homogeneous in appearance under transmission electron microscopy (scale bars, $5 \mu \mathrm{m}$ in left panel and $100 \mathrm{~nm}$ in right panel). (B) The expression levels of HSP70 and CD63 were detected in exosomes isolated from serum samples by Western blot analysis (lane I, serum exosomal pellets; lane 2, exosomes-depleted serum supernatant). $\beta$-Actin was used as loading control. (C) The expression of CD63 was detected in exosomes isolated from serum samples by flow cytometry analysis.

Abbreviations: HSP70, heat shock protein 70; PE, phycoerythrin.
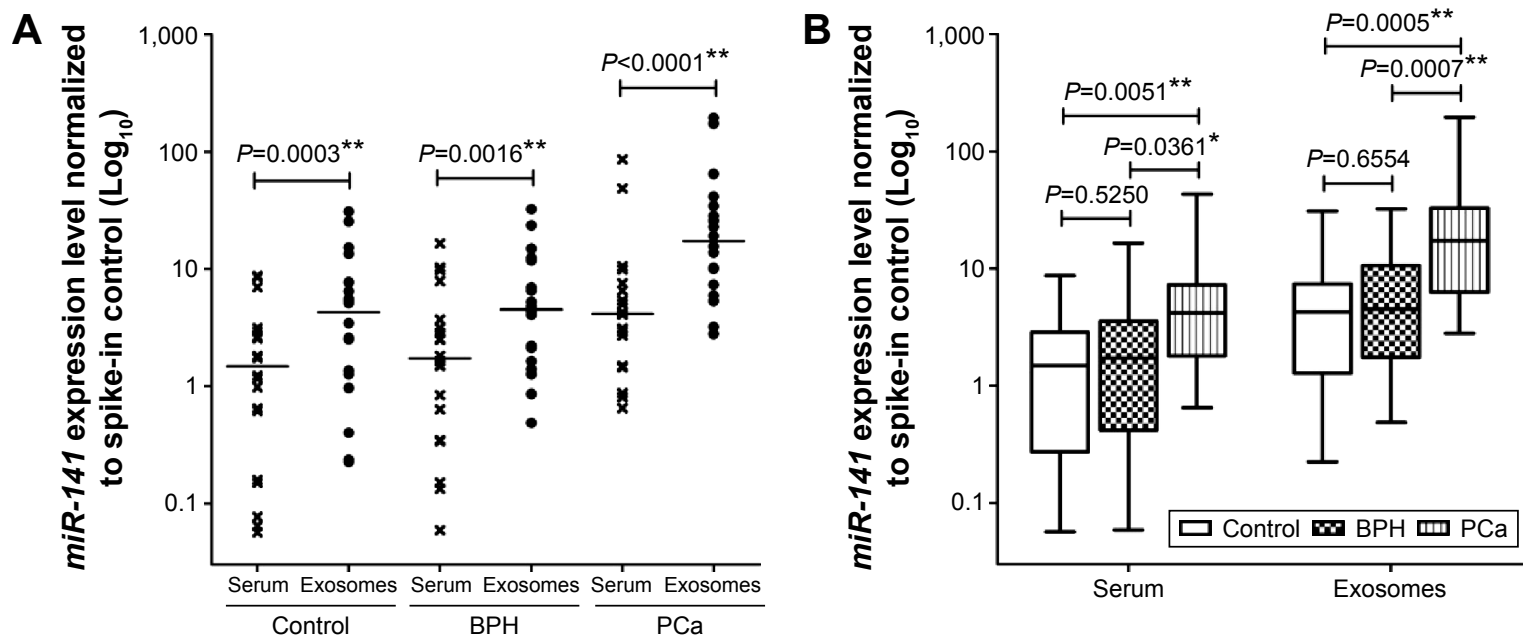

Figure 2 Expression of miR- $/ 4 I$ is significantly higher in exosomes compared with in serum.

Notes: The expression levels of miR-I4I in the serum and in the serum exosomes were measured in 20 patients with PCa, 20 patients with BPH, and 20 healthy volunteers. The expression levels of exosomal miR-I4I were analyzed by quantitative reverse transcription-polymerase chain reaction and normalized to a spike-in control ( $y$-axis, $\left.\log _{10}\right)$. (A) The lines indicate the medians. A Wilcoxon rank sum test was used to determine the statistical significance. (B) The boxes represent the interquartile range. The horizontal lines in the boxes indicate the median levels. Whiskers indicate maximum and minimum values. A Mann-Whitney $U$-test or Kruskal-Wallis test was used to determine the statistical significance. $* P<0.05$, $* * P<0.01$.

Abbreviations: BPH, benign prostate hyperplasia; miR, microRNA; PCa, prostate cancer. 
A

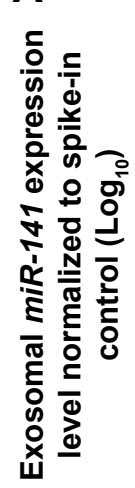

B

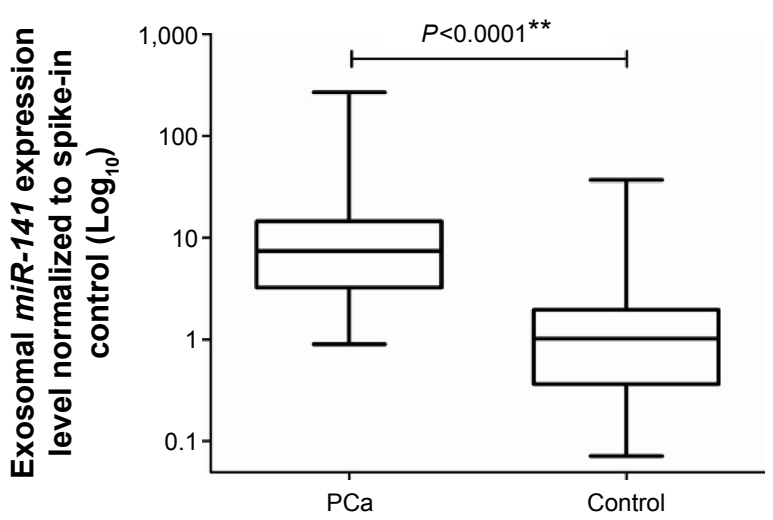

Figure 3 Expression of serum exosomal miR- $/ 4 I$ is significantly higher in patients with $\mathrm{PCa}$.

Notes: (A and B) The expression levels of exosomal miR-I4I are illustrated in serum samples from 5 I patients with PCa and 40 healthy individuals. The exosomal miR-I4I expression levels were analyzed by quantitative reverse transcription-polymerase chain reaction and were normalized against the spike-in control $\left(y\right.$-axis, Log $\left.{ }_{10}\right)$. (B) The boxes represent the interquartile range. The horizontal lines in the boxes indicate the median levels. Whiskers indicate maximum and minimum values. A Mann-Whitney $U$-test was used to determine the statistical significance. $* * P<0.01$.

Abbreviations: miR, microRNA; PCa, prostate cancer.

was to a lesser extent (2.41-fold, $P=0.0361$ and 2.81 -fold, $P=0.0051$, respectively; Figure $2 \mathrm{~B})$. The sensitivity of the circulating miR-141 detection may be improved using exosomes.

\section{Patients with PCa exhibit higher miR-/4I levels in serum exosomes}

In order to understand the potential values of serum exosomal miR-141 in the development and progression of $\mathrm{PCa}$, the exosomal expression levels of miR-141 in the 51 patients with $\mathrm{PCa}$ and the 40 healthy individuals in the follow-up cohort were determined using qRT-PCR (Table 2). As expected, the expression levels of exosomal miR-141 in the PCa group were significantly increased compared with the control group $(P<0.0001 ;$ Figure $3 \mathrm{~A}$ and $\mathrm{B})$.

\section{Exosomal miR-I4I levels correlate with the clinicopathological factors of patients with $\mathrm{PCa}$}

The 51 patients with $\mathrm{PCa}$ were divided into groups of high and low exosomal expression levels of $m i R-141$ using the median $m i R-141$ expression value as the cutoff point. In the high group, $42.31 \%$ of patients were aged $\geq 70$ years, and in the low group, $60 \%$ of patients were aged $\geq 70$ years ( $P=0.085$ ). By contrast, the percentage of patients with PSA values $\geq 10 \mathrm{ng} / \mathrm{mL}$ was higher in the high group compared with the low group $(92.31 \%$ vs $64.00 \%, P=0.024)$. The percentage of patients with a Gleason score $\geq 8$ tumors and patients with T3/T4 diseases was higher in the high group compared with the low group (61.54\% vs $16.00 \%, P<0.0001$; $73.08 \%$ vs $32.00 \%, P=0.0026)$. The majority of patients exhibited metastatic $\mathrm{PCa}$ in the high group compared with the low group (69.23\% vs $8.00 \%, P<0.0001$; Table 3 ). These results revealed that a higher PSA value, higher Gleason score, advanced T-classification, and metastasis were associated with higher serum exosomal expression of miR-141 in patients with $\mathrm{PCa}$.

\section{Exosomal miR-I4I has potential value as a diagnostic biomarker for metastatic $\mathrm{PCa}$} The present study, therefore, further focused on the association between serum exosomal expression of $m i R-141$

Table 3 Association of exosomal miR-/4I expression with the clinicopathological characteristics of patients with $\mathrm{PCa}$

\begin{tabular}{|c|c|c|c|c|}
\hline \multirow[t]{2}{*}{ Characteristic } & \multirow{2}{*}{$\begin{array}{l}\text { Total } \\
\text { number }\end{array}$} & \multicolumn{2}{|c|}{ Exosomal miR-I4I } & \multirow[t]{2}{*}{$P$-value } \\
\hline & & $\begin{array}{l}\text { Low } \\
\text { expression } \\
\text { group }\end{array}$ & $\begin{array}{l}\text { High } \\
\text { expression } \\
\text { group }\end{array}$ & \\
\hline Total number & 51 & 25 & 26 & \\
\hline Age (years) & & & & 0.085 \\
\hline$<70$ & 25 & 10 & 15 & \\
\hline$\geq 70$ & 26 & 15 & II & \\
\hline PSA (ng/mL) & & & & $0.024^{*}$ \\
\hline$<10$ & II & 9 & 2 & \\
\hline$\geq 10$ & 40 & 16 & 24 & \\
\hline Gleason score & & & & $<0.000 I^{* *}$ \\
\hline$<8$ & 31 & 21 & 10 & \\
\hline$\geq 8$ & 20 & 4 & 16 & \\
\hline Clinical stage & & & & $0.0026 * *$ \\
\hline $\mathrm{TI} / \mathrm{T} 2$ & 24 & 17 & 7 & \\
\hline $\mathrm{T} 3 / \mathrm{T} 4$ & 27 & 8 & 19 & \\
\hline Metastasis & & & & $<0.000 I^{* *}$ \\
\hline No & 31 & 23 & 8 & \\
\hline Yes & 20 & 2 & 18 & \\
\hline
\end{tabular}

Notes: $* P<0.05, * * P<0.01$.

Abbreviations: miR, microRNA; PCa, prostate cancer; PSA, prostate-specific antigen. 
and the tumor stage, metastasis, as well as on the potential value of exosomal miR-141 as a diagnostic biomarker for PCa.

First, serum exosomal expression of $m i R-141$ in the patients with different Gleason scores was determined. As shown in Figure 4A, significant differences were observed when patients with a Gleason score $\geq 7$ tumors were compared with the controls $(P<0.0001)$. However, no statistically significant difference was observed between low Gleason score $(\leq 6)$ tumors and the controls $(P=0.0619)$.

A

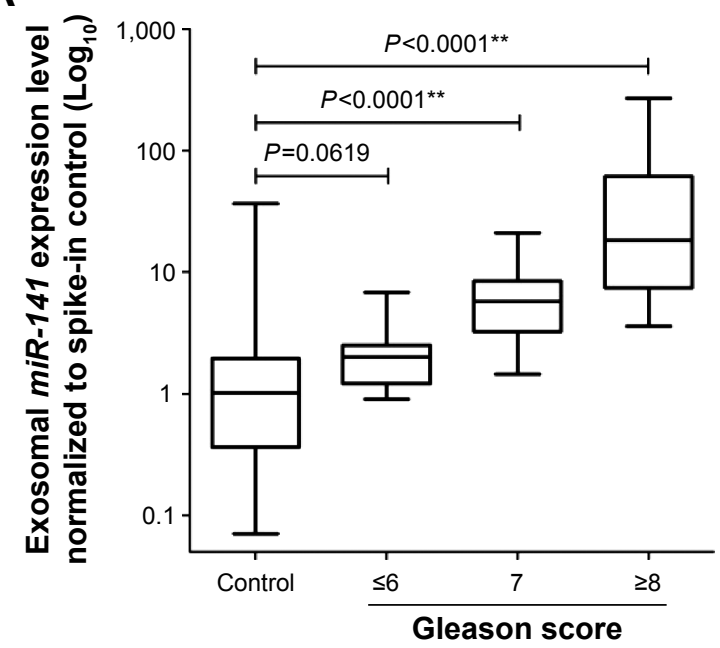

C

Localized PCa vs metastatic PCa (PSA)

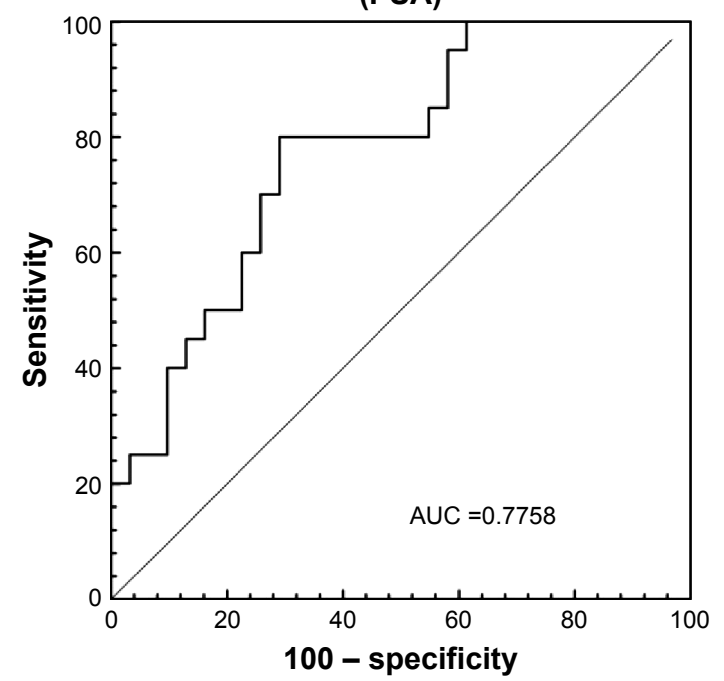

Subsequently, the levels of serum exosomal $m i R-141$ in the 20 patients with metastatic PCa were compared with the 31 patients with localized PCa. The median exosomal $m i R-141$ levels were 21.019 in the metastatic group and 4.449 in the localized group $(P<0.0001)$. Each group was significantly higher compared with the control group $(P<0.0001$; Figure 4B).

Subsequently, ROC curves were generated in order to assess the power of exosomal miR-141 to distinguish the patients with metastatic PCa from the patients with
B

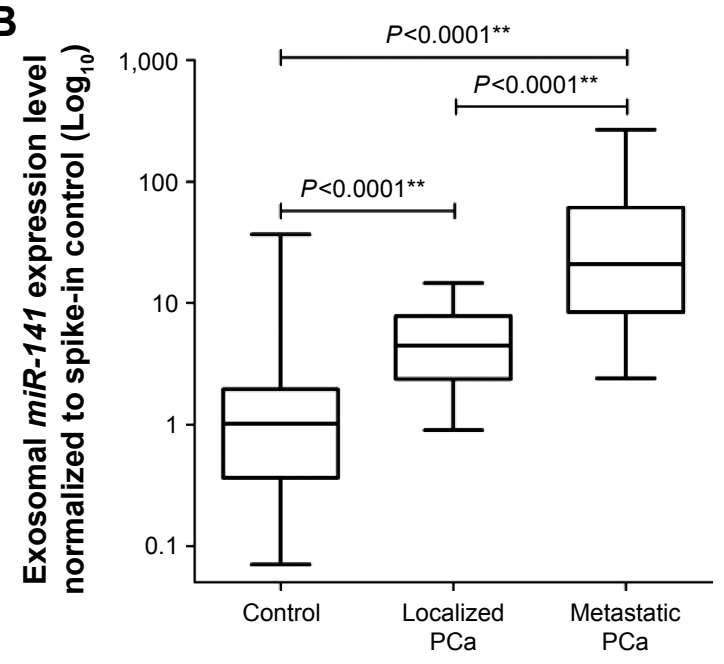

D

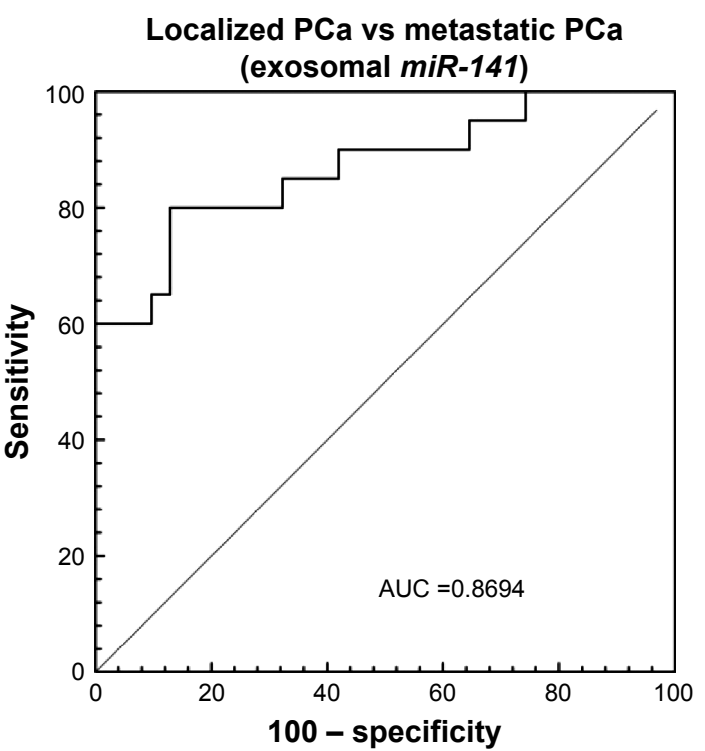

Figure 4 Expression levels of serum exosomal miR-/4I in subgroups of patients with PCa.

Notes: (A) A box plot of serum exosomal miR-I4I levels across the PCa Gleason score. Quantitative reverse transcription-polymerase chain reaction was performed and normalized against the spike-in control $\left(y\right.$-axis, $\left.\log _{10}\right)$. The boxes represent the interquartile range. The horizontal lines in the boxes indicate the median levels. Whiskers indicate maximum and minimum values. A Mann-Whitney U-test or Kruskal-Wallis test was used to determine the statistical significance. (B) The expression levels of serum exosomal miR-/4I were measured in 20 patients with metastatic PCa, 3 I patients with localized PCa, and 40 healthy individuals by quantitative reverse transcription-polymerase chain reaction and were normalized against the spike-in control $\left(y\right.$-axis, $\left.\log _{10}\right)$. The boxes represent the interquartile range. The horizontal lines in the boxes indicate the median levels. Whiskers indicate maximum and minimum values. A Mann-Whitney $U$-test or Kruskal-Wallis test was used to determine the statistical significance. Receiver-operating characteristic analysis of (C) PSA and (D) exosomal miR-/4I was used to differentiate between patients with metastatic and patients with localized PCa. PSA yielded an AUC of 0.7758 with $80 \%$ sensitivity and $70.97 \%$ specificity in discriminating metastatic PCa. Exosomal miR-I 4 I yielded an AUC of 0.8694 with $80 \%$ sensitivity and $87.10 \%$ specificity in discriminating metastatic $P C a$. $* * P<0.01$.

Abbreviations: AUC, area under the curve; miR, microRNA; PCa, prostate cancer; PSA, prostate-specific antigen. 
localized PCa. The analyses revealed that the exosomal $m i R-141$ had a higher AUC (AUC $=0.8694,95 \%$ confidence interval $=0.7625-0.9762)$ compared with the PSA (AUC $=0.7758,95 \%$ confidence interval $=0.6486-0.9030)$. At the cutoff value of 8.362 for the relative expression of exosomal $m i R-141$, the sensitivity was $80 \%$ and the specificity was $87.10 \%$. At the cutoff value of 60.13 for PSA, the sensitivity was $80 \%$ and the specificity was $70.97 \%$ (Figure $4 \mathrm{C}$ and D). The ROC curve indicated that exosomal $m i R-141$ was an improved discriminator to distinguish between patients with metastatic PCa and those with localized PCa.

\section{Discussion}

The RNA sequencing analysis of plasma-derived exosomes revealed that miRNAs were the most abundant exosomal RNA species, comprising $76.20 \%$ of all mappable reads. ${ }^{34}$ In addition, Gallo et $\mathrm{al}^{13}$ indicated that the majority of circulating miRNAs that were detectable in the serum and saliva were concentrated in exosomes and that exosome isolation may improve the sensitivity of miRNA amplification from human biological fluids. A previous study reported that exosomes provide a protective and enriched source of miRNA for biomarker profiling, and the exosomes isolated from serum contained a higher percentage of miRNA compared with the plasma. ${ }^{35}$ In addition, it was demonstrated that serum exosomes may serve as a suitable material to measure circulating miRNA biomarkers for the diagnosis and prognosis of diseases. ${ }^{36,37}$ A previous study reported that a set of circulating exosomal miRNAs can be used as the noninvasive diagnostic biomarker of colon cancer. ${ }^{38}$ Tanaka et $\mathrm{al}^{7}$ confirmed that serum expression of exosomal miR-21 expression was upregulated in patients with esophageal squamous cell carcinoma and was positively correlated with tumor progression and aggressiveness. Taken together, these findings suggest that the serum exosomal miRNAs may be important in noninvasive diagnostic testing. Therefore, the aim of the present study was to determine if exosomes derived from patients with PCa were valuable for cancer diagnosis.

It has been previously reported that the expression levels of $m i R-141$ are correlated with a high Gleason score or metastasis of the patients with PCa. ${ }^{14,27-30}$ However, the comparison of the expression levels of $m i R-141$ in the serum samples and exosomes isolated from the serum of patients with PCa remains to be elucidated. In order to determine whether exosomes are an improved material in comparison with the total serum for miRNA testing, miR-141 was used as an example to compare the miRNA expression levels in the whole serum and the serum exosomes of patients with
PCa, BPH, and healthy volunteers. Furthermore, the potential clinical significance of exosomal $m i R-141$ for the detection of PCa was also assessed.

First, the expression levels of miR-141 were analyzed by qRT-PCR in the whole serum samples and serum exosomal pellets isolated from the same serum in a cohort of 20 patients with $\mathrm{PCa}, 20$ patients with $\mathrm{BPH}$, and 20 healthy control individuals. This revealed that the expression of exosomal $m i R-141$ in patients with $\mathrm{PCa}$ was significantly higher compared with the patients with $\mathrm{BPH}$ and the healthy controls. In addition, significant correlations were observed in the miR-141 expression levels between the whole serum and exosomes, and the expression levels of exosomal miR-141 were all significantly higher compared with the serum circulating $m i R-141$ in the three groups. Therefore, it was suggested that $m i R-141$ was enriched in exosomes, which provides increased sensitivity of detection compared with the whole serum.

In the second validation set, significantly elevated levels of serum exosomal miR-141 were observed in patients with $\mathrm{PCa}$, and were considerably correlated with cancer metastasis. However, no significant difference was observed between a low Gleason score tumor and the controls. It was suggested that serum exosomal miR-141 failed to facilitate early detection of the primary PCa.

However, the ROC curve indicated that, in comparison with PSA, exosomal miR-141 was an improved discriminator to distinguish between patients with metastatic PCa and those with localized PCa. Therefore, it is likely that the serum exosomal miR-141 was more associated with metastatic PCa disease compared with the grading of primary PCa. These observations suggested that serum exosomal miRNAs, including exosomal miR-141, may be useful to predict the risk of metastatic $\mathrm{PCa}$, which is consistent with previous reports regarding circulating $m i R-141$.

However, the role of exosomal miR-141 in PCa progression remains to be elucidated and must be further examined in functional studies. The exact role of $m i R-141$ in cancer requires further investigation, particularly in metastatic PCa.

In conclusion, the present study demonstrated that serum exosomes were a more accurate material compared with the whole serum for measuring circulating miR-141 levels in patients with PCa. Exosomal miR-141 may be used as a valuable noninvasive biomarker for the detection of metastatic PCa. To the best of our knowledge, the present study compared the differences between miRNA analyses using total serum and the serum exosomes for the first time, and 
evaluated the potential clinical significance of serum exosomal miR-141 for the detection of metastatic PCa.

\section{Acknowledgments}

The authors would like to thank Dr Ping Meng, Dr Jun-Hui Liu, and Dr Guang-Feng Zhu for their contribution in clinical samples collection.

\section{Disclosure}

The authors report no conflicts of interest in this work.

\section{References}

1. Siegel RL, Miller KD, Jemal A. Cancer statistics, 2015. CA Cancer J Clin. 2015;65(1):5-29.

2. Chen W, Zheng R, Zeng H, Zhang S, He J. Annual report on status of cancer in China, 2011. Chin J Cancer Res. 2015;27(1):2-12.

3. Wang Y, Guo J, Xu L, et al. Should bone scan be performed in Chinese prostate cancer patients at the time of diagnosis? Urol Int. 2013;91(2): $160-164$.

4. Zhang J, Dhakal IB, Zhao Z, Li L. Trends in mortality from cancers of the breast, colon, prostate, esophagus, and stomach in East Asia: role of nutrition transition. Eur J Cancer Prev. 2012;21(5):480-489.

5. Draisma G, Etzioni R, Tsodikov A, et al. Lead time and overdiagnosis in prostate-specific antigen screening: importance of methods and context. J Natl Cancer Inst. 2009;101(6):374-383.

6. Manterola L, Guruceaga E, Gallego Perez-Larraya J, et al. A small noncoding RNA signature found in exosomes of GBM patient serum as a diagnostic tool. Neuro Oncol. 2014;16(4):520-527.

7. Tanaka Y, Kamohara H, Kinoshita K, et al. Clinical impact of serum exosomal microRNA-21 as a clinical biomarker in human esophageal squamous cell carcinoma. Cancer. 2013;119(6):1159-1167.

8. Bala S, Petrasek J, Mundkur S, et al. Circulating microRNAs in exosomes indicate hepatocyte injury and inflammation in alcoholic, drug-induced, and inflammatory liver diseases. Hepatology. 2012;56(5): 1946-1957.

9. Colombo M, Raposo G, Thery C. Biogenesis, secretion, and intercellular interactions of exosomes and other extracellular vesicles. Annu Rev Cell Dev Biol. 2014;30:255-289.

10. Regev-Rudzki N, Wilson DW, Carvalho TG, et al. Cell-cell communication between malaria-infected red blood cells via exosome-like vesicles. Cell. 2013;153(5):1120-1133.

11. Shimbo K, Miyaki S, Ishitobi H, et al. Exosome-formed synthetic microRNA-143 is transferred to osteosarcoma cells and inhibits their migration. Biochem Biophys Res Commun. 2014;445(2):381-387.

12. Ge Q, Zhou Y, Lu J, Bai Y, Xie X, Lu Z. miRNA in plasma exosome is stable under different storage conditions. Molecules. 2014;19(2): $1568-1575$.

13. Gallo A, Tandon M, Alevizos I, Illei GG. The majority of microRNAs detectable in serum and saliva is concentrated in exosomes. PLoS One. 2012;7(3):e30679.

14. Brase JC, Johannes M, Schlomm T, et al. Circulating miRNAs are correlated with tumor progression in prostate cancer. Int JCancer. 2011;128(3): 608-616.

15. Kuner R, Brase JC, Sultmann H, Wuttig D. microRNA biomarkers in body fluids of prostate cancer patients. Methods. 2013;59(1):132-137.

16. Nguyen HC, Xie W, Yang M, et al. Expression differences of circulating microRNAs in metastatic castration resistant prostate cancer and low-risk, localized prostate cancer. Prostate. 2013;73(4):346-354.

17. Lin HM, Castillo L, Mahon KL, et al. Circulating microRNAs are associated with docetaxel chemotherapy outcome in castration-resistant prostate cancer. Br J Cancer. 2014;110(10):2462-2471.
18. Huang X, Yuan T, Liang M, et al. Exosomal miR-1290 and miR-375 as prognostic markers in castration-resistant prostate cancer. Eur Urol. 2015;67(1):33-41.

19. Zhou X, Wang Y, Shan B, et al. The downregulation of miR-200c/141 promotes ZEB1/2 expression and gastric cancer progression. Med Oncol. 2015;32(1):428.

20. Du Y, Xu Y, Ding L, et al. Down-regulation of miR-141 in gastric cancer and its involvement in cell growth. J Gastroenterol. 2009;44(6): $556-561$.

21. Zhu ZM, Xu YF, Su QJ, et al. Prognostic significance of microRNA141 expression and its tumor suppressor function in human pancreatic ductal adenocarcinoma. Mol Cell Biochem. 2014;388(1-2):39-49.

22. Xu F, He H, Huang W, et al. Decreased expression of MicroRNA-200 family in human breast cancer is associated with lymph node metastasis. Clin Transl Oncol. Epub 2015 Jul 23.

23. O'Day E, Lal A. MicroRNAs and their target gene networks in breast cancer. Breast Cancer Res. 2010;12(2):201.

24. Yu XY, Zhang Z, Liu J, Zhan B, Kong CZ. MicroRNA-141 is downregulated in human renal cell carcinoma and regulates cell survival by targeting CDC25B. Onco Targets Ther. 2013;6:349-354.

25. Nakada C, Matsuura K, Tsukamoto Y, et al. Genome-wide microRNA expression profiling in renal cell carcinoma: significant down-regulation of miR-141 and miR-200c. J Pathol. 2008;216(4):418-427.

26. Xue J, Niu YF, Huang J, et al. miR-141 suppresses the growth and metastasis of HCC cells by targeting E2F3. Tumour Biol. 2014;35(12): 12103-12107.

27. Mitchell PS, Parkin RK, Kroh EM, et al. Circulating microRNAs as stable blood-based markers for cancer detection. Proc Natl Acad Sci U S A. 2008;105(30):10513-10518.

28. Yaman Agaoglu F, Kovancilar M, Dizdar Y, et al. Investigation of miR-21, miR-141, and miR-221 in blood circulation of patients with prostate cancer. Tumour Biol. 2011;32(3):583-588.

29. Bryant RJ, Pawlowski T, Catto JW, et al. Changes in circulating microRNA levels associated with prostate cancer. Br J Cancer. 2012;106(4): 768-774.

30. Selth LA, Townley S, Gillis JL, et al. Discovery of circulating microRNAs associated with human prostate cancer using a mouse model of disease. Int J Cancer. 2012;131(3):652-661.

31. Ravindranath MH, Muthugounder S, Presser N, et al. Endogenous immune response to gangliosides in patients with confined prostate cancer. Int J Cancer. 2005;116(3):368-377.

32. Lasser C, Eldh M, Lotvall J. Isolation and characterization of RNAcontaining exosomes. J Vis Exp. 2012;(59):e3037.

33. Livak KJ, Schmittgen TD. Analysis of relative gene expression data using real-time quantitative PCR and the 2(-Delta Delta C(T)) Method. Methods. 2001;25(4):402-408.

34. Huang X, Yuan T, Tschannen M, et al. Characterization of human plasma-derived exosomal RNAs by deep sequencing. BMC Genomics. 2013;14:319.

35. Cheng L, Sharples RA, Scicluna BJ, Hill AF. Exosomes provide a protective and enriched source of miRNA for biomarker profiling compared to intracellular and cell-free blood. J Extracell Vesicles. 2014; 3:23743.

36. Alegre E, Sanmamed MF, Rodriguez C, Carranza O, Martin-Algarra S, Gonzalez A. Study of circulating microRNA-125b levels in serum exosomes in advanced melanoma. Arch Pathol Lab Med. 2014;138(6): $828-832$.

37. Wang H, Hou L, Li A, Duan Y, Gao H, Song X. Expression of serum exosomal microRNA-21 in human hepatocellular carcinoma. Biomed Res Int. 2014;2014:864894.

38. Ogata-Kawata H, Izumiya M, Kurioka D, et al. Circulating exosomal microRNAs as biomarkers of colon cancer. PLoS One. 2014;9(4): e92921. 


\section{Supplementary material}

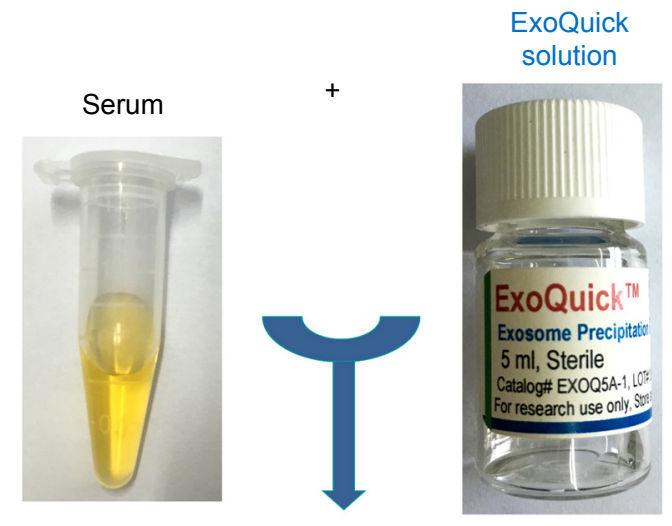

Incubation $\left(4^{\circ} \mathrm{C}, 30\right.$ minutes $)$

(One-step precipitation)

Centrifugation $(1,500 \times g, 30$ minutes $)$

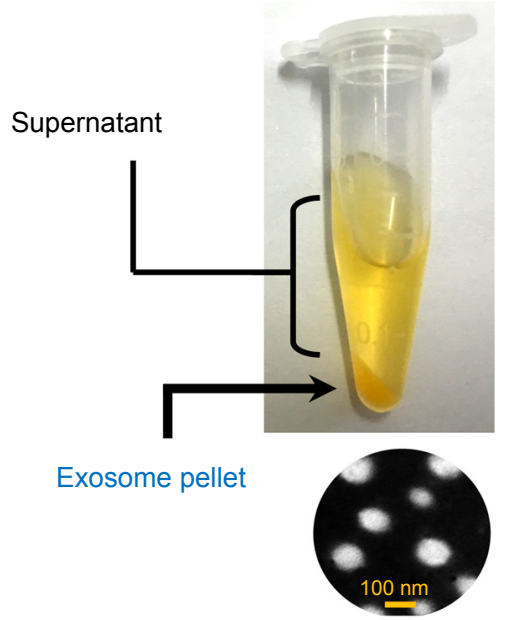

Figure SI Flow chart for exosomal isolation.

Notes: Exosomes were extracted from serum sample using ExoQuick Exosome Precipitation Solution (System Biosciences, Mountain View, CA, USA). A quarter volume of ExoQuck solution was added to serum and the samples were incubated at $4^{\circ} \mathrm{C}$ for 30 minutes, followed by centrifugation at 1,500× $\mathrm{g}$ for 30 minutes. The final pellets, containing exosomes, were collected for characterization and RNA isolation.

\section{Publish your work in this journal}

OncoTargets and Therapy is an international, peer-reviewed, open access journal focusing on the pathological basis of all cancers, potential targets for therapy and treatment protocols employed to improve the management of cancer patients. The journal also focuses on the impact of management programs and new therapeutic agents and protocols on patient perspectives such as quality of life, adherence and satisfaction. The manuscript management system is completely online and includes a very quick and fair peer-review system, which is all easy to use. Visit http://www.dovepress.com/testimonials.php to read real quotes from published authors. 